\title{
Pharmacotherapeutic Options for Managing Neuropathic Pain: A Systematic Review and Meta-Analysis
}

\author{
Giulia Di Stefano (D, Andrea Di Lionardo, Giuseppe Di Pietro, Giorgio Cruccu, \\ and Andrea Truini
}

Department of Human Neuroscience, Sapienza University of Rome, Rome, Italy

Correspondence should be addressed to Giulia Di Stefano; giulia.distefano@uniroma1.it

Received 13 October 2020; Accepted 20 April 2021; Published 26 April 2021

Academic Editor: Marina De Tommaso

Copyright ( 2021 Giulia Di Stefano et al. This is an open access article distributed under the Creative Commons Attribution License, which permits unrestricted use, distribution, and reproduction in any medium, provided the original work is properly cited.

\begin{abstract}
Despite an increasing number of available therapies, the treatment of neuropathic pain remains a major issue. Systematic reviews and meta-analyses indicate that only a minority of patients with neuropathic pain have an adequate response to pharmacological treatment and that most drugs have dose-limiting side effects. We conducted a systematic review and meta-analysis of randomised controlled trials published in the last five years. We searched for relevant papers within PubMed, EMBASE, the Cochrane Database of Systematic Reviews, and the Clinical Trials database (ClinicalTrials.gov). Two authors independently selected studies for inclusion, data extraction, and bias assessment. We identified 39 randomised controlled trials and included 16 in the metaanalysis. Trial outcomes were generally modest even for first-line drugs such as tricyclic antidepressants, serotonin-noradrenaline reuptake inhibitors, and gabapentinoids. Many drugs acting on new pain targets are currently under development. Clinical data are currently available for sodium channel isoform-specific antagonists, anti-nerve growth factor molecules, and fatty acid amide hydrolase inhibitors.
\end{abstract}

\section{Introduction}

Despite an increasing number of available therapies, the treatment of neuropathic pain remains a major issue. Systematic reviews and meta-analyses indicate that only a minority of patients with neuropathic pain have an adequate response to pharmacological treatment and that most drugs have dose-limiting side effects $[1,2]$.

In 2015, the Neuropathic Pain Special Interest Group (NeuPSIG) of the International Association for the Study of Pain (IASP) conducted a systematic review and meta-analysis that included 229 randomised double-blind trials testing oral and topical pharmacotherapy for neuropathic pain [3]. This analysis led to a strong GRADE recommendation for the use of tricyclic antidepressants (TCAs), serotonin-noradrenaline reuptake inhibitor (SNRI) antidepressants, pregabalin, gabapentin, and gabapentin/enacarbil XR and the proposal of these drugs as first-line treatments. In addition, the analysis provided a weak recommendation for the use of lidocaine patches, capsaicin patches, and tramadol, the proposal of these drugs as second-line treatments, a weak recommendation for the use of strong opioids (particularly oxycodone and morphine) and botulinum toxin A (BTX-A), and the proposal of these drugs as third-line treatments. Data for tapentadol and drug combinations were inconclusive. A weak recommendation was provided against cannabinoid use in neuropathic pain [3].

In the last few years, new medical treatments for neuropathic pain have been tested, including compounds acting on new pain targets [1].

We conducted a systematic review and meta-analysis of randomised controlled trials (RCTs) of all pharmacological 
treatments for neuropathic pain published in 2015 or later in order to analyse the efficacy and safety of drugs tested in the last five years and provide an update to the 2015 NeuPSIG meta-analysis.

\section{Search Process}

Systematic literature review was performed according to the Preferred Reporting Items for Systematic Reviews and MetaAnalyses (PRISMA) statements. We searched for relevant papers within PubMed, EMBASE, and the Cochrane Database of Systematic Reviews and considered studies published in peer-reviewed journals between January 2015 and July 2020. Search terms were related to pharmacological treatment of neuropathic pain. The primary search was supplemented by a secondary search using the bibliographies of retrieved articles. RCTs involving at least 10 patients were considered, and the search was limited to English language publications. Studies published only as abstracts were excluded. We checked ClinicalTrial.gov in order to include studies currently in progress. The target population was patients of any age with neuropathic pain according to the IASP definition (i.e., pain caused by a lesion or disease of the somatosensory nervous system) and related to different aetiologies. Mixed pain (e.g., cancer pain and low back pain) and conditions such as fibromyalgia and atypical facial pain were not included in the analysis.

The review process was performed independently by two reviewers (Figure 1). The authors independently assessed the quality of the individual trials during data extraction. Two independent authors assessed the studies in terms of methodological quality using the five-point Oxford Quality Scale [4]. A minimum score of 2 out of 5 (randomised and double-blind studies) was required for inclusion.

\section{Statistics}

The number needed to treat (NNT) for $50 \%$ pain intensity reduction (alternatively, 30\% pain reduction) was considered as the primary effect measure in the meta-analysis. The $95 \%$ confidence interval (CI) for NNT values was calculated as the reciprocal value of the $95 \% \mathrm{CI}$ for the absolute risk difference using normal approximation. Studies testing combination therapies were excluded from the meta-analysis. In dosefinding studies, data from subgroups treated with low doses were not included in the meta-analysis. Pooled NNT was calculated by considering a fixed-effects model based on the Mantel-Haenszel formula. The pooled variance of the studies, used to evaluate the confidence interval, was computed according to the Greenland-Robins formula. We assessed heterogeneity among studies by calculating the heterogeneity index $I^{2}$ [5] on the basis of the pooled $\chi 2$. When the overall heterogeneity $I^{2}$ exceeded a given threshold (30\%), a randomeffects model was considered, using the DerSimonian and Laird method for variance. Statistical analysis was performed using GraphPad Prism 8 software.

\section{Results}

We identified 436 RCTs in patients with neuropathic pain (Figure 1). After abstract screening, 85 full texts were assessed for eligibility. We excluded 17 studies that tested the effects of drugs in patients with mixed or nociceptive pain and two studies that involved a small number of patients (less than 10). We assessed the risk of bias in the remaining 66 trials through the five-point Oxford Quality Scale [4]. We excluded 27 trials with a score less than 2. Thirty-nine RCTs were included in the systematic review (Tables 1-7), and 16 RCTs that provided NNT values were included in the metaanalysis (Table 8).

4.1. Antidepressants. Five RCTs globally involving 486 patients tested the effects of antidepressants, including venlafaxine, duloxetine, amitriptyline, and imipramine, in patients with neuropathic pain related to different aetiologies (Table 1) [6-10]. In two RCTs, the effect of duloxetine $60 \mathrm{mg}$ and amitriptyline $10 \mathrm{mg}$ was compared to that of gabapentin $900 \mathrm{mg}[6,8]$. In the study by Majdinasab et al., both duloxetine and gabapentin were effective in relieving pain in patients with painful diabetic neuropathy; though gabapentin showed an earlier effect, it had more side effects. Conversely, duloxetine had better medication compliance. At the end of a 6-week trial conducted by Brown et al., amitriptyline and gabapentin significantly decreased pain intensity scores without significant differences between the two drugs in terms of their effects on pain reduction and the frequency of adverse events. In the study by Farshchian et al., 156 patients with chemotherapy-induced peripheral neuropathy were randomly assigned to one of three pharmacotherapy groups: venlafaxine, duloxetine, or placebo. Neuropathic pain decreased significantly in both the venlafaxine and duloxetine groups as compared with placebo. Duloxetine was more effective than venlafaxine in reducing pain. No severe side effects were reported in the two groups.

Two studies involving 192 patients with neuropathic pain related to spinal cord injury and painful neuropathy were included in the metanalysis $[9,10]$. The authors tested the effects of venlafaxine XR and imipramine in comparison with placebo. The effect of venlafaxine $\mathrm{XR}$ on neuropathic pain was similar to that of placebo [9]. Imipramine was tested both in monotherapy and in combination with pregabalin [10]. A $50 \%$ pain relief was reported by $20 \%$ of patients treated with imipramine. The combined NNT using a random-effects model was $12.41(4.07 ; \infty ;-11.83)$ (test for heterogeneity: $\left.\quad \chi^{2}=2.1 ; \quad \mathrm{d} f=1 ; \quad P=0.15 ; \quad I^{2}=51.28\right)$ (Figure 2(a)).

4.2. Gabapentinoids. Nine RCTs globally involving 3903 patients tested the effect of gabapentinoids on neuropathic pain related to different aetiologies, including diabetic polyneuropathy, postherpetic neuralgia, and radiotherapyrelated neuropathic pain (Table 2) [10-18]. 


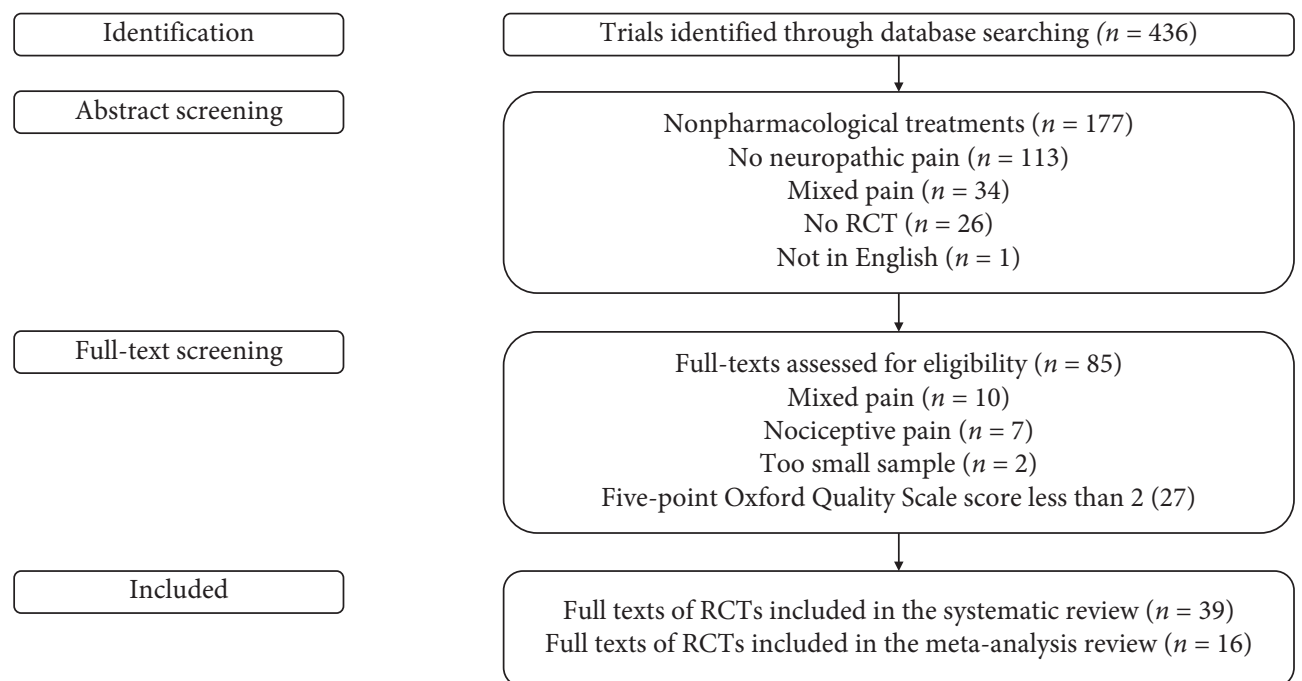

FIGURE 1: Flowchart of the search process.

TABLE 1: Randomized, double-blind, controlled trials testing the effect of antidepressants.

\begin{tabular}{|c|c|c|c|c|c|c|c|}
\hline Study & Active drug & Control & $\begin{array}{l}\text { Sample } \\
\text { size }\end{array}$ & NP condition & $\begin{array}{l}\text { Outcome } \\
\text { measure }\end{array}$ & $\begin{array}{c}\text { Pain } \\
\text { outcome }\end{array}$ & Adverse events \\
\hline $\begin{array}{l}\text { Majdinasab } \\
\text { et al. [6] }\end{array}$ & Duloxetine $60 \mathrm{mg}$ & $\begin{array}{l}\text { Gabapentin } \\
900 \mathrm{mg}\end{array}$ & 104 & $\mathrm{DPN}$ & 0-100 VAS & $\begin{array}{l}\text { Positive } \\
\text { with both } \\
\text { drugs }\end{array}$ & $\begin{array}{l}\text { Anxiety (2\%) and } \\
\text { sleeplessness }(2 \%)\end{array}$ \\
\hline $\begin{array}{l}\text { Farshchian } \\
\text { et al. [7] }\end{array}$ & $\begin{array}{c}\text { Venlafaxine } \\
37.5 \mathrm{mg} ; \\
\text { duloxetine } 30 \mathrm{mg}\end{array}$ & Placebo & 156 & CIPN & $\begin{array}{l}0-3 \\
\text { neuropathic } \\
\text { pain grade }\end{array}$ & Positive & $\begin{array}{l}\text { Venlafaxine: nausea (12\%), } \\
\text { constipation }(7 \%) \text {, and } \\
\text { insomnia }(7 \%) \text {; duloxetine: } \\
\text { dizziness }(11 \%) \text {, fatigue } \\
(10 \%) \text {, and headache }(4 \%)\end{array}$ \\
\hline $\begin{array}{l}\text { Brown et al. } \\
\text { [8] }\end{array}$ & $\begin{array}{l}\text { Amitriptyline } \\
10 \mathrm{mg}\end{array}$ & $\begin{array}{l}\text { Gabapentin } \\
900 \mathrm{mg}\end{array}$ & 34 & $\begin{array}{l}\text { Different NP } \\
\text { conditions }\end{array}$ & $\begin{array}{l}\text { 0-10 CAS } \\
\text { score }\end{array}$ & $\begin{array}{l}\text { Positive } \\
\text { with both } \\
\text { drugs }\end{array}$ & Not reported \\
\hline $\begin{array}{l}\text { Richards } \\
\text { et al. [9] }\end{array}$ & $\begin{array}{c}\text { Venlafaxine XR } \\
75-225 \mathrm{mg}\end{array}$ & Placebo & 123 & SCI & 0-10 NRS & Negative & Not reported \\
\hline $\begin{array}{l}\text { Holbech et al. } \\
{[10]}\end{array}$ & $\begin{array}{l}\text { Imipramine } \\
75 \mathrm{mg}\end{array}$ & Placebo & 69 & $\begin{array}{c}\text { Painful } \\
\text { polyneuropathy }\end{array}$ & 0-10 NRS & Positive & $\begin{array}{c}\text { Dizziness }(10 \%) \text {, sweating } \\
(20 \%) \text { dry mouth }(22 \%) \text {, and } \\
\text { paraesthesia }(10 \%)\end{array}$ \\
\hline
\end{tabular}

NP: neuropathic pain; DPN: diabetic painful neuropathy; CIPN: chemotherapy-induced peripheral neuropathy; SCI: spinal cord injury; NRS: numerical rating scale; CAS: colour analogue scale; VAS: visual analogue scale.

Four RCTs testing the effect of pregabalin $[10,12,14,15]$ and two RCTs testing the effect of mirogabalin $[11,13]$ were included in the meta-analysis. The combined NNT using a random-effects model was $8.40(4.85 ; 31.15)$ (test for heterogeneity: $\left.\quad \chi^{2}=24.2 ; \quad \mathrm{d} f=5 ; \quad P=0.0002 ; \quad I^{2}=79.32 \%\right)$ (Figure 2(b)).

The most common side effects with pregabalin included dizziness, somnolence, oedema, and weight gain. In patients treated with mirogabalin, the most reported side effects were nasopharyngitis, somnolence, and dizziness (Table 2).

Two RCTs testing the effect of mirogabalin on central neuropathic pain and diabetic peripheral neuropathic pain are currently ongoing (ClinicalTrials.gov Identifier: NCT03901352; NCT04094662). The combination of gabapentin and trazodone is currently being tested in painful diabetic neuropathy (ClinicalTrials.gov Identifier: NCT03749642) (Lipone et al., 2020).
4.3. Lidocaine. Five RCTs globally involving 670 patients tested the effect of lidocaine in patients with localised peripheral neuropathic pain (Table 3) [19-23]. Three RCTs $[19,20,23]$ testing the effect of the lidocaine patch $5 \%$ in localised peripheral neuropathic pain were included in the meta-analysis. The combined NNT using a fixed-effects model was $12.73(6.94 ; 76.79)$ (test for heterogeneity: $\chi^{2}=2.9 ; \mathrm{d} f=2 ; P=0.240 ; I^{2}=29.86 \%$ ) (Figure $2(\mathrm{~d})$ ). The most common side effects included mild skin reactions (Table 4).

4.4. Opioids. Four RCTs globally involving 215 patients tested the effect of opioids in different neuropathic pain conditions, including postherpetic neuralgia, diabetic peripheral neuropathy, and central pain related to multiple sclerosis (Table 4) [24-27]. 
TABLE 2: Randomized, double-blind, controlled trials testing the effect of gabapentinoids.

\begin{tabular}{|c|c|c|c|c|c|c|c|c|c|}
\hline Study & Active drug & Control & $\begin{array}{l}\text { Sample } \\
\text { size }\end{array}$ & \multicolumn{2}{|c|}{ NP condition } & \multicolumn{2}{|c|}{$\begin{array}{l}\text { Outcome } \\
\text { measure }\end{array}$} & $\begin{array}{c}\text { Pain } \\
\text { outcome }\end{array}$ & Adverse events (>10\%) \\
\hline $\begin{array}{l}\text { Baba et al. } \\
{[11]}\end{array}$ & $\begin{array}{l}\text { Mirogabalin 15/ } \\
\text { 20/30 mg }\end{array}$ & Placebo & 834 & \multicolumn{2}{|c|}{ DPN } & \multicolumn{2}{|c|}{$0-10$ NRS } & Positive & $\begin{array}{c}\text { Nasopharyngitis }(16.4 \%) \text {, } \\
\text { somnolence }(14.5 \%) \text {, and } \\
\text { dizziness }(18 \%)\end{array}$ \\
\hline $\begin{array}{l}\text { Jiang et al. } \\
{[12]}\end{array}$ & $\begin{array}{l}\text { Pregabalin } \\
300-600 \mathrm{mg}\end{array}$ & Placebo & 128 & \multicolumn{2}{|c|}{$\begin{array}{l}\text { Radiotherapy- } \\
\text { induced NP }\end{array}$} & \multicolumn{2}{|c|}{$0-10$ NRS } & Positive & $\begin{array}{c}\text { Dizziness }(18.8 \%) \text { and } \\
\text { somnolence }(20.3 \%)\end{array}$ \\
\hline $\begin{array}{l}\text { Kato et al. } \\
{[13]}\end{array}$ & $\begin{array}{l}\text { Mirogabalin 15/ } \\
\text { 20/30 mg }\end{array}$ & Placebo & 765 & \multicolumn{2}{|c|}{ PHN } & \multicolumn{2}{|c|}{$0-10$ NRS } & Positive & $\begin{array}{c}\text { Somnolence }(23.9 \%) \text {, } \\
\text { nasopharyngitis }(12.9) \text {, and } \\
\text { dizziness }(15.5 \%)\end{array}$ \\
\hline $\begin{array}{l}\text { Huffman } \\
\text { et al. [14] }\end{array}$ & $\begin{array}{l}\text { Pregabalin CR } \\
82.5-660 \mathrm{mg}\end{array}$ & Placebo & 413 & \multicolumn{2}{|c|}{ PHN } & \multicolumn{2}{|c|}{ NPS } & Positive & $\begin{array}{c}\text { Dizziness (17.1\%) and somnolence } \\
(11.4 \%)\end{array}$ \\
\hline Liu et al. [15] & $\begin{array}{l}\text { Pregabalin } \\
300 \mathrm{mg}\end{array}$ & Placebo & 220 & \multicolumn{2}{|c|}{ PHN } & \multicolumn{2}{|c|}{$0-10$ NRS } & Positive & Dizziness (24.3\%) \\
\hline $\begin{array}{l}\text { Merante } \\
\text { et al. [16] }\end{array}$ & $\begin{array}{l}\text { Mirogabalin 15/ } \\
\text { 20/30 mg }\end{array}$ & Placebo & 452 & \multicolumn{2}{|c|}{ DPN } & \multicolumn{2}{|c|}{ PGIC, BPI } & Positive & $\begin{array}{l}\text { Dizziness }(15.8 \%) \text { and } \\
\text { somnolence }(12.3 \%)\end{array}$ \\
\hline $\begin{array}{l}\text { Raskin et al. } \\
\text { [17] }\end{array}$ & $\begin{array}{l}\text { Pregabalin } \\
150-300 \mathrm{mg}\end{array}$ & Placebo & 301 & \multicolumn{2}{|c|}{ DPN } & \multicolumn{2}{|c|}{$0-10 \mathrm{NRS}$} & Negative & Dizziness (10.3) \\
\hline $\begin{array}{l}\text { Freeman } \\
\text { et al. }[18]\end{array}$ & $\begin{array}{l}\text { Gabapentin } \\
1800 \mathrm{mg}\end{array}$ & Placebo & 721 & \multicolumn{2}{|c|}{ PHN } & \multicolumn{2}{|c|}{ 0-100 NRS } & Positive & Not reported \\
\hline $\begin{array}{l}\text { Holbech } \\
\text { et al. [10] }\end{array}$ & $\begin{array}{l}\text { Pregabalin } \\
300 \mathrm{mg}\end{array}$ & Placebo & 69 & \multicolumn{2}{|c|}{$\begin{array}{c}\text { Painful } \\
\text { polyneuropathy }\end{array}$} & \multicolumn{2}{|c|}{$0-10 \mathrm{NRS}$} & Positive & $\begin{array}{l}\text { Dizziness }(16 \%) \text {, oedema }(16 \%), \\
\text { and weight gain }(14 \%)\end{array}$ \\
\hline \multicolumn{10}{|c|}{$\begin{array}{l}\text { NP: neuropathic pain; DPN: diabetic painful neuropathy; PHN: postherpetic neuralgia; NRS: numerical rating scale; NPS: neuropathic pain scale; PGIC: } \\
\text { patient global impression of change; BPI: brief pain inventory. }\end{array}$} \\
\hline Study & Active drug & Control & $\begin{array}{l}\text { Sample } \\
\text { size }\end{array}$ & $\begin{array}{c}\mathrm{NP} \\
\text { condition }\end{array}$ & $\begin{array}{l}\text { Outc } \\
\text { meas }\end{array}$ & & $\begin{array}{c}\text { Pain } \\
\text { outcome }\end{array}$ & & Adverse events \\
\hline $\begin{array}{l}\text { Palladini et al. } \\
\text { [19] }\end{array}$ & $\begin{array}{l}\text { Lidocaine } 5 \% \\
12 \mathrm{~h} / \text { day }\end{array}$ & Placebo & 363 & $\begin{array}{l}\text { Localized } \\
\text { NP }\end{array}$ & $0-10$ & IRS & Negative & e gastroe & $\begin{array}{l}\text { ain }(7.3 \%), \text { headache }(5.6 \%) \text {, } \\
\text { nteritis }(2.8 \%) \text {, and application site } \\
\quad \text { pruritus }(2.8 \%)\end{array}$ \\
\hline $\begin{array}{l}\text { Pickering } \\
\text { et al. [20] }\end{array}$ & $\begin{array}{l}\text { Lidocaine } 5 \% \\
12 \mathrm{~h} / \text { day }\end{array}$ & Placebo & 36 & $\begin{array}{l}\text { Localized } \\
\text { NP }\end{array}$ & $0-10$ & IRS & Positive & Skin a & $\begin{array}{l}\text { and subcutaneous tissue disorders } \\
\qquad(25.0 \%)\end{array}$ \\
\hline Kim et al. [21] & $\begin{array}{l}\text { Lidocaine IV } \\
\quad(3 \mathrm{mg} / \mathrm{kg})\end{array}$ & Placebo & 42 & $\begin{array}{l}\text { PHN or } \\
\text { CPRS }\end{array}$ & $0-10$ & IRS & Positive & & hest discomfort in one patient \\
\hline Liu et al. [22] & $\begin{array}{l}\text { Lidocaine IV } \\
\quad(5 \mathrm{mg} / \mathrm{kg})\end{array}$ & Placebo & 183 & PHN & VA & & Negative & $\begin{array}{l}\text { Dizzi } \\
\text { headac }\end{array}$ & $\begin{array}{l}\text { ness }(21.1 \%) \text {, dry mouth }(15.6 \%) \text {, } \\
\text { che }(6.7 \%) \text {, and drowsiness }(5.6 \%)\end{array}$ \\
\hline $\begin{array}{l}\text { Demant et al. } \\
\text { [23] }\end{array}$ & $\begin{array}{l}\text { Lidocaine } 5 \% \\
12 \mathrm{~h} / \text { day }\end{array}$ & Placebo & 46 & $\begin{array}{l}\text { Localized } \\
\text { NP }\end{array}$ & $0-10$ & IRS & Positive & & Mild skin reaction $(21 \%)$ \\
\hline
\end{tabular}

NP: neuropathic pain; NRS: numerical rating scale; PHN: postherpetic neuralgia; CPRS: complex regional pain syndrome; VAS: visual analogue scale.

TABLE 4: Randomized, double-blind, controlled trials testing the effect of opioids.

\begin{tabular}{|c|c|c|c|c|c|c|c|}
\hline Study & Active drug & Control & $\begin{array}{l}\text { Sample } \\
\text { size }\end{array}$ & $\begin{array}{c}\mathrm{NP} \\
\text { condition }\end{array}$ & $\begin{array}{c}\text { Outcome } \\
\text { measure }\end{array}$ & $\begin{array}{c}\text { Pain } \\
\text { outcome }\end{array}$ & Adverse events \\
\hline $\begin{array}{l}\text { Gavin et al. } \\
{[24]}\end{array}$ & $\begin{array}{l}\text { Oxycodone patch } \\
23.6 \mathrm{mg} / 72 \text { hours }\end{array}$ & Placebo & 28 & PHN & 0-10 NRS & Negative & $\begin{array}{c}\text { Application site irritation } \\
(18.5 \%) \text {, infections }(7.4 \%), \\
\text { respiratory disorders }(7.4 \%) \text {, } \\
\text { gastrointestinal disorders } \\
(3.7 \%) \text {, musculoskeletal, and } \\
\text { connective tissue disorders } \\
(3.7 \%)\end{array}$ \\
\hline Rigo et al. [25] & $\begin{array}{l}\text { Methadone } 3 \mathrm{mg} \\
\text { ketamine } 30 \mathrm{mg}\end{array}$ & $\begin{array}{l}\text { Methadone } 3 \mathrm{mg} \\
\text { or ketamine } \\
30 \mathrm{mg}\end{array}$ & 42 & $\begin{array}{l}\text { Refractory } \\
\text { NP }\end{array}$ & VAS & Negative * & $\begin{array}{l}\text { Somnolence }(46 \%) \text {, nausea } \\
(23 \%) \text {, vomiting }(15 \%) \text {, and } \\
\text { constipation }(8 \%)\end{array}$ \\
\hline $\begin{array}{l}\text { Simpson and } \\
\text { Wlodarczyk } \\
{[26]}\end{array}$ & $\begin{array}{l}\text { Buprenorphine } \\
\text { patch }<40 \mathrm{mg} / \mathrm{h}\end{array}$ & Placebo & 93 & $\mathrm{DPN}$ & 0-10 NRS & Negative & $\begin{array}{l}\text { Nausea }(43.0 \%) \text { and } \\
\text { constipation }(31.2 \%)\end{array}$ \\
\hline $\begin{array}{l}\text { Gilron et al. } \\
\text { [27] }\end{array}$ & $\begin{array}{l}\text { Morphine } 10 \mathrm{mg} \\
\text { nortriptyline } \\
10 \mathrm{mg}\end{array}$ & $\begin{array}{l}\text { Nortriptyline } \\
10 \mathrm{mg} \text { or } \\
\text { morphine } 10 \mathrm{mg}\end{array}$ & 52 & $\begin{array}{l}\text { Peripheral } \\
\text { NP }\end{array}$ & $0-10$ NRS & Positive & $\begin{array}{c}\text { Dry mouth }(57.5 \%), \\
\text { constipation }(42.5 \%) \text {, and } \\
\text { somnolence }(20 \%)\end{array}$ \\
\hline
\end{tabular}


TABLE 5: Randomized, double-blind, controlled trials testing the effect of cannabinoids.

\begin{tabular}{|c|c|c|c|c|c|c|c|}
\hline Study & Active drug & Control & $\begin{array}{l}\text { Sample } \\
\text { size }\end{array}$ & $\begin{array}{c}\mathrm{NP} \\
\text { condition }\end{array}$ & $\begin{array}{l}\text { Outcome } \\
\text { measure }\end{array}$ & $\begin{array}{c}\text { Pain } \\
\text { outcome }\end{array}$ & Adverse events (>10\%) \\
\hline $\begin{array}{l}\text { Schimrigk et al. } \\
\text { [28] }\end{array}$ & $\begin{array}{l}\text { Dronabinol } \\
7.5-15.0 \mathrm{mg}\end{array}$ & Placebo & 238 & MS & $0-10$ NRS & Negative & Dizziness (17.4\%) \\
\hline $\begin{array}{l}\text { van } \\
\text { Amerongen } \\
\text { et al. [29] }\end{array}$ & D9-THC 16 mg & Placebo & 24 & MS & $0-10$ NRS & Negative & $\begin{array}{c}\text { Dizziness }(58.3 \%) \text {, headache }(50 \%) \text {, } \\
\text { muscular weakness }(33.3 \%) \text {, somnolence } \\
(25 \%) \text {, paraesthesia }(16.7 \%) \text {, tremor } \\
(16.7 \%) \text {, tinnitus }(16.7 \%), \text { psychiatric/ } \\
\text { mood }(33.3 \%) \text {, fatigue }(16.7 \%) \text {, and dry } \\
\text { mouth }(16.7 \%)\end{array}$ \\
\hline $\begin{array}{l}\text { Wilsey et al. } \\
{[30]}\end{array}$ & $\begin{array}{l}\text { D9-THC } \\
2.9-6.7 \%\end{array}$ & Placebo & 42 & SCI & $0-10$ NRS & Positive & Not reported \\
\hline $\begin{array}{l}\text { Turcotte et al. } \\
{[31]}\end{array}$ & $\begin{array}{l}\text { Nabilone } 2 \mathrm{mg} \text {; } \\
\text { gabapentin } \\
\geq 1.800 \mathrm{mg}\end{array}$ & Placebo & 15 & $\begin{array}{l}\text { MS- } \\
\text { related NP }\end{array}$ & VAS & Positive & $\begin{array}{l}\text { Dizziness (62.5\%), drowsiness (50\%), and } \\
\text { dry mouth (50\%) }\end{array}$ \\
\hline $\begin{array}{l}\text { Wallace et al. } \\
{[32]}\end{array}$ & D9-THC $1 / 4 / 7 \%$ & Placebo & 16 & $\mathrm{DPN}$ & VAS & Positive & $\begin{array}{c}\text { Euphoria (100\%) and somnolence } \\
(73.3 \%)\end{array}$ \\
\hline
\end{tabular}

NP: neuropathic pain; MS: multiple sclerosis; DPN: diabetic painful neuropathy; NRS: numerical rating scale; SCI: spinal cord injury; VAS: visual analog scale.

In the study by Gavin et al., the transdermal oxycodone patch was compared with placebo in patients with postherpetic neuralgia [24]. The authors found that the oxycodone patch did not significantly reduce pain, though patients reporting high levels of paraesthesia showed a trend toward improved pain reduction.

In the study by Simpson and Wlodarczyk, the buprenorphine patch up to $40 \mu \mathrm{g} / \mathrm{h}$ was tested in comparison with placebo in patients with diabetic peripheral neuropathy [26]. A high proportion of patients did not complete the study due to adverse events, and the primary endpoint was not reached.

Two RCTs compared combination therapy with monotherapy $[25,27]$. In the study by Rigo et al., the effect of oral methadone combined with oral ketamine was compared to that of monotherapy [25]. A significant pain improvement was observed in the ketamine alone group as compared with both the methadone and methadone/ketamine groups. In the study by Gilron et al., the nortriptyline-morphine combination was compared with each monotherapy [27]. The study showed superior efficacy of combination treatment as compared with monotherapy. The most frequent adverse events included constipation, dry mouth, and somnolence (Table 5).

4.5. Cannabinoids. Five RCTs globally involving 335 patients tested the effect of cannabinoids in patients with central neuropathic pain related to multiple sclerosis and spinal cord injury and in patients with pain related to diabetic peripheral neuropathy (Table 5) [28-32]. In the two RCTs involving patients with multiple sclerosis $[28,29]$, tetrahydrocannabinol (THC) did not significantly reduce pain in comparison with placebo.

The effect of nabilone $2 \mathrm{mg}$ combined with gabapentin was compared with placebo in patients with central neuropathic pain related to multiple sclerosis [31]. Pain decrease was statistically greater in the nabilone vs. placebo study group. Nabilone was well tolerated, with dizziness and drowsiness being the most frequently reported side effects.
Two RCTs [30, 32] were included in the meta-analysis. The combined NNT using a random-effects model was 2.96 $(1.78 ; 8.77)$ (test for heterogeneity: $\chi^{2}=1.7 ; \mathrm{d} f=1 ; P=0.191$; $\mathrm{I}^{2}=41.41 \%$ ) (Figure $2(\mathrm{e})$ ). The most frequently reported side effects included dizziness, somnolence, muscle weakness, paraesthesia, tremor, tinnitus, psychiatric/mood disorders, fatigue, and dry mouth (Table 6).

The effect of acutely administered cannabidiol/THC is currently being tested in HIV-related neuropathic pain (ClinicalTrials.gov Identifier: NCT03099005).

4.6. Sodium Channel Blockers. Four RCTs globally involving 319 patients tested the effect of selective sodium channel blockers in different neuropathic pain conditions, including trigeminal neuralgia, postherpetic neuralgia, and painful polyneuropathy (Table 6) [33-36]. A double-blind placebocontrolled randomised withdrawal phase IIa trial tested the effect of a new voltage- and frequency-dependent sodium channel blocker selective for the sodium channel 1.7 (Nav1.7) subtype in 67 patients with trigeminal neuralgia [35]. A 21-day open-label treatment period using BIIB074 $150 \mathrm{mg}$ three times/day was followed by randomisation into a double-blind 28-day treatment phase with either placebo or BIIB074 in only those patients who showed a successful treatment response within the final week. Although the primary endpoint of treatment failure was not significantly lower in the BIIB074 group as compared with the placebo group, significant treatment differences as compared with placebo were found in secondary endpoints, including time to treatment failure, number of paroxysms, and average daily pain score. A phase III trial is currently ongoing, though recruitment has not yet started (ClinicalTrials.gov Identifier: NCT03637387).

A novel selective Nav1.7 sodium channel blocker (PF05089771) was also tested in 135 patients with diabetic peripheral neuropathy [34]. Although a trend toward a reduction in the weekly average pain score in the PF05089771 treatment group was observed, this was not statistically significant when compared with placebo. 
TABLE 6: Randomized, double-blind, controlled trials testing the effect of sodium channel blockers.

\begin{tabular}{|c|c|c|c|c|c|c|c|}
\hline Reference & Active drug & Control & $\begin{array}{l}\text { Sample } \\
\text { size }\end{array}$ & $\begin{array}{c}\mathrm{NP} \\
\text { condition }\end{array}$ & $\begin{array}{l}\text { Outcome } \\
\text { measure }\end{array}$ & $\begin{array}{c}\text { Pain } \\
\text { outcome }\end{array}$ & Adverse events \\
\hline $\begin{array}{l}\text { de Greef et al. } \\
\text { [33] }\end{array}$ & $\begin{array}{l}\text { Lacosamide } \\
\quad 400 \mathrm{mg}\end{array}$ & Placebo & 47 & $\begin{array}{l}\text { Nav1.7- } \\
\text { related SFN }\end{array}$ & $0-10$ NRS & Positive & $\begin{array}{l}\text { Dizziness }(41.7) \text {, nausea }(25 \%) \text {, headache } \\
(25 \%) \text {, fatigue }(20.8 \%) \text {, tremor }(20.8 \%) \text {, } \\
\text { somnolence }(16.7 \%) \text {, epigastric } \\
\text { discomfort }(16.7 \%) \text {, memory impairment } \\
(12.5 \%) \text {, and pruritus }(12.5 \%)\end{array}$ \\
\hline $\begin{array}{l}\text { Mc Donnell } \\
\text { et al. [34] }\end{array}$ & $\begin{array}{l}\text { PF-05089771 } \\
300 \mathrm{mg}\end{array}$ & Placebo & 135 & DPN & 0-10 NRS & Negative & $\begin{array}{c}\text { Constipation }(5 \%) \text {, back pain }(1 \%) \\
\text { headache }(1 \%) \text {, and pollakiuria }(1 \%)\end{array}$ \\
\hline $\begin{array}{l}\text { Zakrzewska } \\
\text { et al. [35] }\end{array}$ & $\begin{array}{c}\text { BIIB074 } \\
450 \mathrm{mg}\end{array}$ & Placebo & 67 & $\mathrm{TN}$ & $\begin{array}{l}\text { Number of } \\
\text { treatment } \\
\text { failures }\end{array}$ & Negative & $\begin{array}{c}\text { Headache }(19 \%) \text {, dizziness }(9 \%), \\
\text { dyspepsia }(6 \%) \text {, diarrhoea }(6 \%), \\
\text { abdominal pain }(6 \%) \text {, and fatigue }(6 \%)\end{array}$ \\
\hline Price et al. [36] & $\begin{array}{l}\text { TV-45070 } \\
\text { ointment }\end{array}$ & Placebo & 70 & PHN & $\begin{array}{l}\text { Mean daily } \\
\text { pain score }\end{array}$ & Negative & $\begin{array}{c}\text { Application site pain }(15.9 \%) \text {, application } \\
\text { site pruritus }(12.7 \%) \text {, and infections } \\
(17.5 \%)\end{array}$ \\
\hline
\end{tabular}

NP: neuropathic pain; SFN: small-fibre neuropathy; DPN: diabetic painful neuropathy; TN: trigeminal neuralgia; PHN: postherpetic neuralgia; NRS: numerical rating scale.

TABLE 7: Randomized, double-blind, controlled trials testing the effect of other drugs.

\begin{tabular}{|c|c|c|c|c|c|c|c|}
\hline Study & Active drug & Control & $\begin{array}{l}\text { Sample } \\
\text { size }\end{array}$ & NP condition & $\begin{array}{l}\text { Outcome } \\
\text { measure }\end{array}$ & $\begin{array}{c}\text { Pain } \\
\text { outcome }\end{array}$ & Adverse events \\
\hline $\begin{array}{l}\text { Kulkantrakorn } \\
\text { et al. [37] }\end{array}$ & $\begin{array}{c}\text { Capsaicin } \\
0.075 \%\end{array}$ & Placebo & 42 & $\mathrm{DPN}$ & VAS & Negative & $\begin{array}{c}\text { Skin reaction (50\%), burning sensation } \\
(41.7 \%) \text {, and erythema }(11.1 \%)\end{array}$ \\
\hline $\begin{array}{l}\text { Kerckhove et al. } \\
\text { [38] }\end{array}$ & $\begin{array}{l}\text { Ethosuximide } \\
1500 \mathrm{mg}\end{array}$ & Placebo & 114 & $\begin{array}{l}\text { Peripheral } \\
\text { NP }\end{array}$ & $0-10$ NRS & Negative & $\begin{array}{c}\text { Dyspepsia }(39 \%) \text {, headache }(32 \%) \text {, and } \\
\text { dizziness }(20 \%) .\end{array}$ \\
\hline Hor et al. [39] & $\begin{array}{l}\text { Tocotrienols } \\
400 \mathrm{mg}\end{array}$ & Placebo & 300 & DPN & TSS & Negative & Not reported \\
\hline $\begin{array}{l}\text { Bradford et al. } \\
{[40]}\end{array}$ & $\begin{array}{l}\text { ASP8477 } \\
20-60 \mathrm{mg}\end{array}$ & Placebo & 132 & $\begin{array}{l}\text { DPN and } \\
\text { PHN }\end{array}$ & $0-10 \mathrm{NRS}$ & Negative & $\begin{array}{c}\text { Allergic dermatitis }(2.7 \%) \text {, increased } \\
\text { appetite }(2.7 \%) \text {, and musculoskeletal } \\
\text { stiffness }(2.7 \%)\end{array}$ \\
\hline $\begin{array}{l}\text { Andresen et al. } \\
{[41]}\end{array}$ & PEA-um $600 \mathrm{mg}$ & Placebo & 73 & SCI & 0-10 NRS & Negative & $\begin{array}{c}\text { Urinary tract infection (1\%), paralytic } \\
\text { ileus (1\%), cholecystolithiasis, and } \\
\text { fungus infection }(1 \%)\end{array}$ \\
\hline Wang et al. [42] & $\begin{array}{l}\text { Fulranumab 1-3- } \\
\qquad 10 \mu \mathrm{g}\end{array}$ & Placebo & & $\begin{array}{l}\text { PHN and } \\
\text { PTN }\end{array}$ & $0-10$ NRS & Negative & $\begin{array}{c}\text { PHN: arthralgia }(21 \%) \text {, osteoarthritis } \\
(21 \%) \text {, back pain }(11 \%) \text {, oedema }(11 \%) \text {, } \\
\text { diarrhoea }(11 \%) \text {, anaemia }(11 \%), \\
\text { influenza }(11 \%) \text {, and urinary tract } \\
\text { infection }(11 \%) \text {, PTN: sinusitis }(17 \%), \\
\text { headache }(13 \%) \text {, and carpal tunnel } \\
\text { syndrome }(13 \%)\end{array}$ \\
\hline $\begin{array}{l}\text { Bramson et al. } \\
{[43]}\end{array}$ & $\begin{array}{l}\text { Tanezumab sc } \\
20 \mu \mathrm{g}\end{array}$ & Placebo & 73 & DPN & 0-10 NRS & Positive & $\begin{array}{c}\text { Arthralgia }(18.4 \%) \text { and pain in the } \\
\text { extremity }(10.5 \%)\end{array}$ \\
\hline $\begin{array}{l}\text { Bramson et al. } \\
{[43]}\end{array}$ & $\begin{array}{l}\text { Tanezumab iv } \\
50-200 \mu \mathrm{g} / \mathrm{kg}\end{array}$ & Placebo & 96 & PHN & 0-10 NRS & Negative & Headache $(12.5 \%)$ \\
\hline Ahmed et al. [44] & $\begin{array}{l}\text { Topical } \\
\text { diclofenac } 1.5 \%\end{array}$ & Placebo & 35 & $\begin{array}{l}\text { Different NP } \\
\text { conditions }\end{array}$ & VAS & Positive & Not reported \\
\hline
\end{tabular}

NP: neuropathic pain; DPN: diabetic painful neuropathy; PHN: postherpetic neuralgia; NRS: numerical rating scale; SCI: spinal cord injury; VAS: visual analog scale. PTN: posttraumatic neuropathy.

A novel topical sodium channel inhibitor (TV-45070) was compared with placebo in 70 patients with postherpetic neuralgia [36]. Although this study had a negative primary endpoint, it found a remarkable analgesic response in the subpopulation with the R1150W polymorphism (63\% responders vs. $35 \%$ of wild-type carriers).

de Greef et al. tested the effect of lacosamide in 47 patients with Nav1.7-mutation-related small-fibre neuropathy [33]. In $58.3 \%$ of patients receiving lacosamide, mean average pain decreased by at least 1 point, compared to $21.7 \%$ in the placebo group.

The combined NNT using a random-effects model was $4.64(2.59 ; 22.40)$ (test for heterogeneity: $\chi^{2}=3.7 ; \mathrm{d} f=3$; $P=0.160 ; I^{2}=45.46 \%$ ) (Figure $2(\mathrm{c})$ ).

The effect of lacosamide on peripheral neuropathic pain with and without the irritable nociceptor phenotype is currently being tested (ClinicalTrials.gov Identifier: NCT03777956). 
TABle 8: Number needed to treat.

\begin{tabular}{|c|c|c|c|}
\hline Study and drug classes & Drug & NNT & ARR \\
\hline \multicolumn{4}{|l|}{ Antidepressants } \\
\hline Richards [9] & Venlafaxine XR $225 \mathrm{mg}$ & $38(5.34 ; \infty ;-4.15)$ & $0.027(-0.24 ; 0.19)$ \\
\hline Holbech [10] & Imipramine $75 \mathrm{mg}$ & $7(4,00 ; 32.51)$ & $0.14(0.03 ; 0.25)$ \\
\hline Pooled & & $13(4.07 ; \infty ;-11.83)$ & $0.08(-0.09 ; 0.25)$ \\
\hline \multicolumn{4}{|l|}{ Gabapentinoids } \\
\hline Baba [11] & Mirogabalin $30 \mathrm{mg}$ & $8(4.87 ; 28.56)$ & $0.12(0.04 ; 0.20)$ \\
\hline Kato [13] & Mirogabalin $30 \mathrm{mg}$ & $18(32,31 ; \infty ;-6.77)$ & $0.06(-0.15 ; 0.03)$ \\
\hline Jiang [12] & Pregabalin 300-600 & $5(2.80 ; 13.73)$ & $0.22(0.09 ; 0.35)$ \\
\hline Huffman [14] & Pregabalin CR $82.5-660 \mathrm{mg}$ & $5(3.69 ; 9.10)$ & $0.19(0.12 ; 0.27)$ \\
\hline Liu [15] & Pregabalin $300 \mathrm{mg}$ & $5(2.97 ; 13.54)$ & $0.21(0.08 ; 0.34)$ \\
\hline Holbech [10] & Pregabalin $300 \mathrm{mg}$ & $17(6.42 ; \infty ;-27.42)$ & $0.06(-0.04 ; 0.16)$ \\
\hline Pooled & & $9(4.85 ; 31.15)$ & $0.12(0.03 ; 0.21)$ \\
\hline \multicolumn{4}{|l|}{ Cannabinoids } \\
\hline Wilsey [30] & D9-THC $2.9-6.7 \%$ & $2(1.64-4.17)$ & $0.43(0.24 ; 0.61)$ \\
\hline Wallace [32] & D9-THC $1-4-7 \%$ & $5(2.03 ; \infty ;-8.53)$ & $0.19(-0.12 ; 0.49)$ \\
\hline Pooled & & $3(1.78 ; 8.77)$ & $0.34(0.11 ; 0.563)$ \\
\hline Pickering [20] & Lidocaine patch $5 \%$ & $3(1.53 ; 73.83)$ & $0.33(0.01 ; 0.65)$ \\
\hline Palladini [19] & Lidocaine patch $5 \%$ & $16(7.21 ; \infty ;-70.24)$ & $0.06(-0.01 ; 0.14)$ \\
\hline Demant [23] & Lidocaine patch $5 \%$ & $20(6.06 ;-15.41)$ & $0.05(-0.07 ; 0.17)$ \\
\hline Pooled & & $13(6.94 ; 76.80)$ & $0.08(-0.01 ; 0.16)$ \\
\hline \multicolumn{4}{|l|}{ Sodium channel blockers } \\
\hline de Greef [33] & Lacosamide $400 \mathrm{mg}$ & $8(3,40 ; \infty ;-21,00)$ & $0.12(-0.05 ; 0.29)$ \\
\hline Zakrzewska [35] & BIIB074 $450 \mathrm{mg}$ & $2(1.31 ; 6.72)$ & $0.46(0.15 ; 0.77)$ \\
\hline Price [36] & TV -45070 & $6(2.60 ; \infty ;-41.3)$ & $0.18(-0.02 ; 0.38)$ \\
\hline Pooled & & $5(2.59 ; 22.40)$ & $0.22(0.05 ; 0.39)$ \\
\hline
\end{tabular}

4.7. Other Drugs. Eight trials testing other drugs in a total of 865 patients were included in the systematic review (Table 7) [37-44].

A randomised double-blinded crossover placebo-controlled trial tested the efficacy and safety of $0.075 \%$ capsaicin lotion in 42 patients with painful diabetic neuropathy [37]. Intention-totreat analysis showed no significant improvement in pain control with capsaicin lotion as compared with placebo for all pain measures.

The effect of ethosuximide was compared with placebo in patients with peripheral neuropathic pain [38]. The study was suspended during interim analysis due to the high number of adverse events in the active treatment group.

Oral mixed tocotrienols were tested in 300 patients with painful diabetic peripheral neuropathy [39]. Mixed tocotrienols $400 \mathrm{mg} /$ day for 1 year did not reduce overall neuropathic symptoms. Tocotrienols were relatively well tolerated, with a safety profile comparable to that of placebo.

A phase IIa trial assessed the analgesic effect and safety of ASP8477, a fatty acid amide hydrolase inhibitor, in 132 patients with painful diabetic peripheral neuropathy and postherpetic neuralgia [40]. ASP8477 was well tolerated but did not demonstrate a significant treatment difference as compared with placebo.

Andresen et al. tested the effect of ultramicronised palmitoylethanolamide (PEA-um) in 73 patients with neuropathic pain related to spinal cord injury [41]. No difference in mean pain intensity between PEA-um and placebo was found. PEA-um was not associated with more adverse effects than placebo.
Two RCTs tested the effect of monoclonal antibodies in neuropathic pain treatment $[42,43]$. In a phase II trial, fulranumab was compared with placebo in patients with postherpetic neuralgia and painful posttraumatic neuropathy [42]. Fulranumab did not significantly reduce pain in comparison with placebo. The most common adverse events included sinusitis, carpal tunnel syndrome, headache, and arthralgia. The study was prematurely suspended due to potential safety concerns with this drug class (rapidly progressive osteoarthritis).

Subcutaneous and intravenous tanezumab was compared with placebo in patients with diabetic peripheral neuropathy and postherpetic neuralgia [43]. Only diabetic patients reported greater mean pain reduction with tanezumab than with placebo. In patients with postherpetic neuralgia, only the highest tanezumab dose $(200 \mu \mathrm{g} / \mathrm{kg})$ reduced pain, but treatment differences were not significant. The most common side effects included arthralgia and headache.

Topical diclofenac (1.5\%) was compared with placebo in 28 patients with neuropathic pain [44]. After 2 weeks of topical application, subjects treated with diclofenac showed a significantly lower overall visual pain score compared with the placebo group.

Two randomised placebo-controlled trials testing the effect of EMA 401 (an oral angiotensin type-II antagonist) in postherpetic neuralgia (ClinicalTrials.gov Identifier: NCT03094195) and painful diabetic neuropathy (ClinicalTrials.gov Identifier: NCT03297294) were prematurely suspended due to preclinical toxicity data. 
Richards et al. 2015;

NNT: 38 (5.34; $\infty ;-4.15)$

Holbech et al. 2015;

NNT: 7 (4.00; 32.51)

Pooled NNT: 12.41

$(4.07 ; \infty ;-11.83)$

Antidepressants

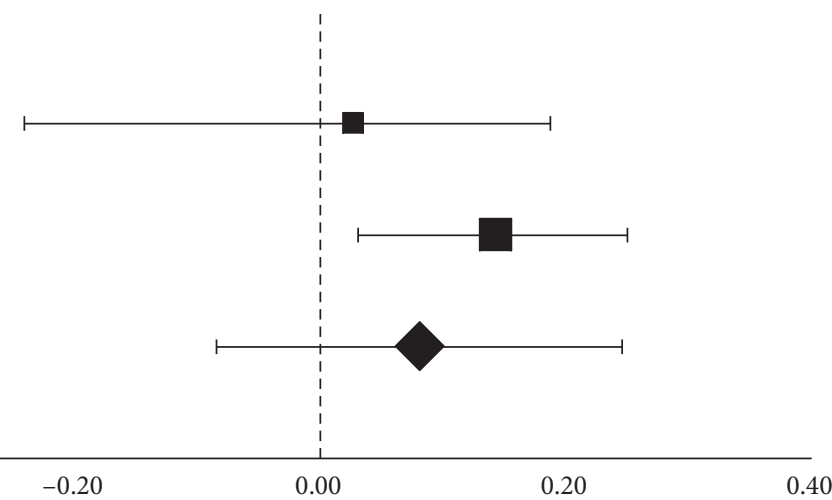

(a)

Gabapentinoids

Baba et al. 2019; NNT: 8

(4.87; 28.56)

Kato et al. 2019; NNT: 18

$(32.31 ; \infty ;-6.77)$

Jiang et al. 2018; NNT: 5

$(2.80 ; 13.73)$

Huffman et al. 2017;

NNT: 5 (3.69; 9.10)

Liu et al. 2015; NNT: 5

(2.97; 13.54)

Holbeck et al. 2015;

NNT: 17 (6.42; $\infty ;-27.42)$

Pooled NNT: 8.40 (4.85;

31.15)

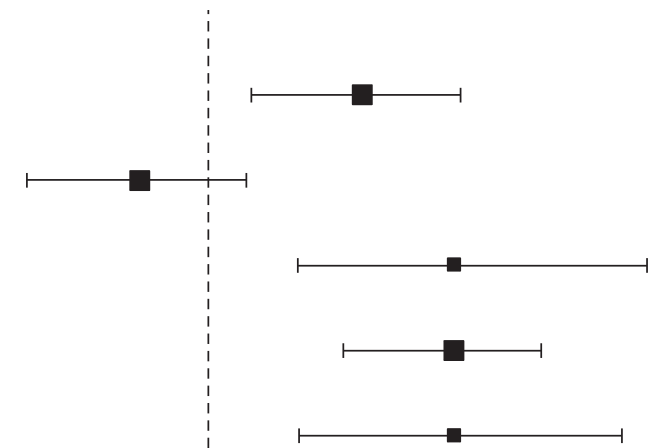

de Greef et al. 2019;

NNT: $8(3.40 ; \infty ;-21.00)$

Zakrzewska et al. 2017;

NNT: 2 (1.31; 6.72)

Price et al. 2017; NNT: $6(2.60 ; \infty ;-41.30)$

Pooled NNT: 5 (2.59;

22.40)

$-0.20$

(b)

Selective sodium channel blockers

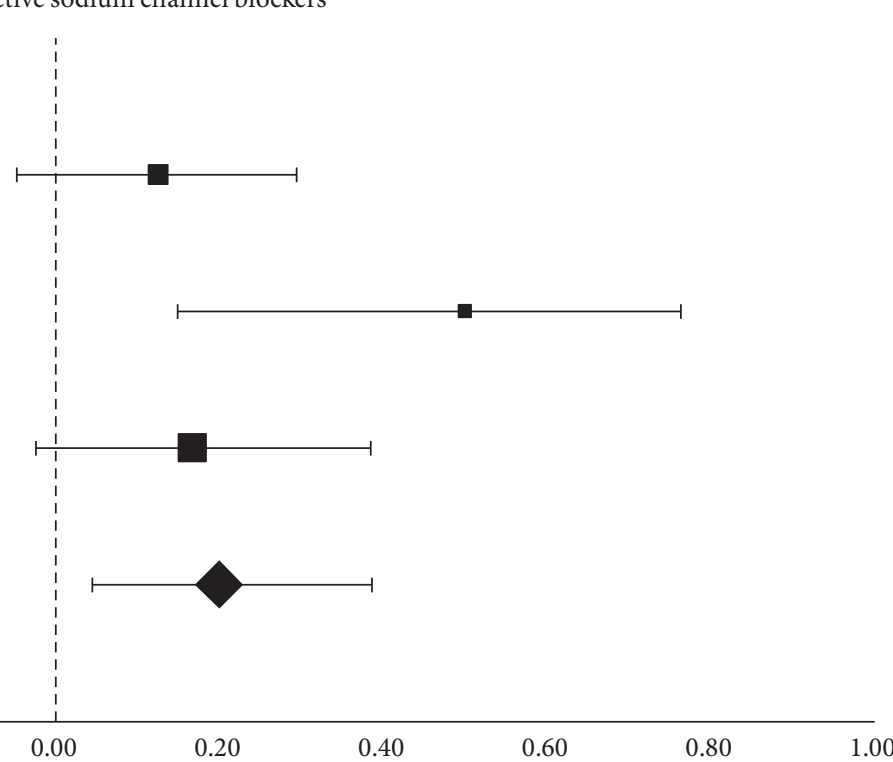

(c)

Figure 2: Continued. 


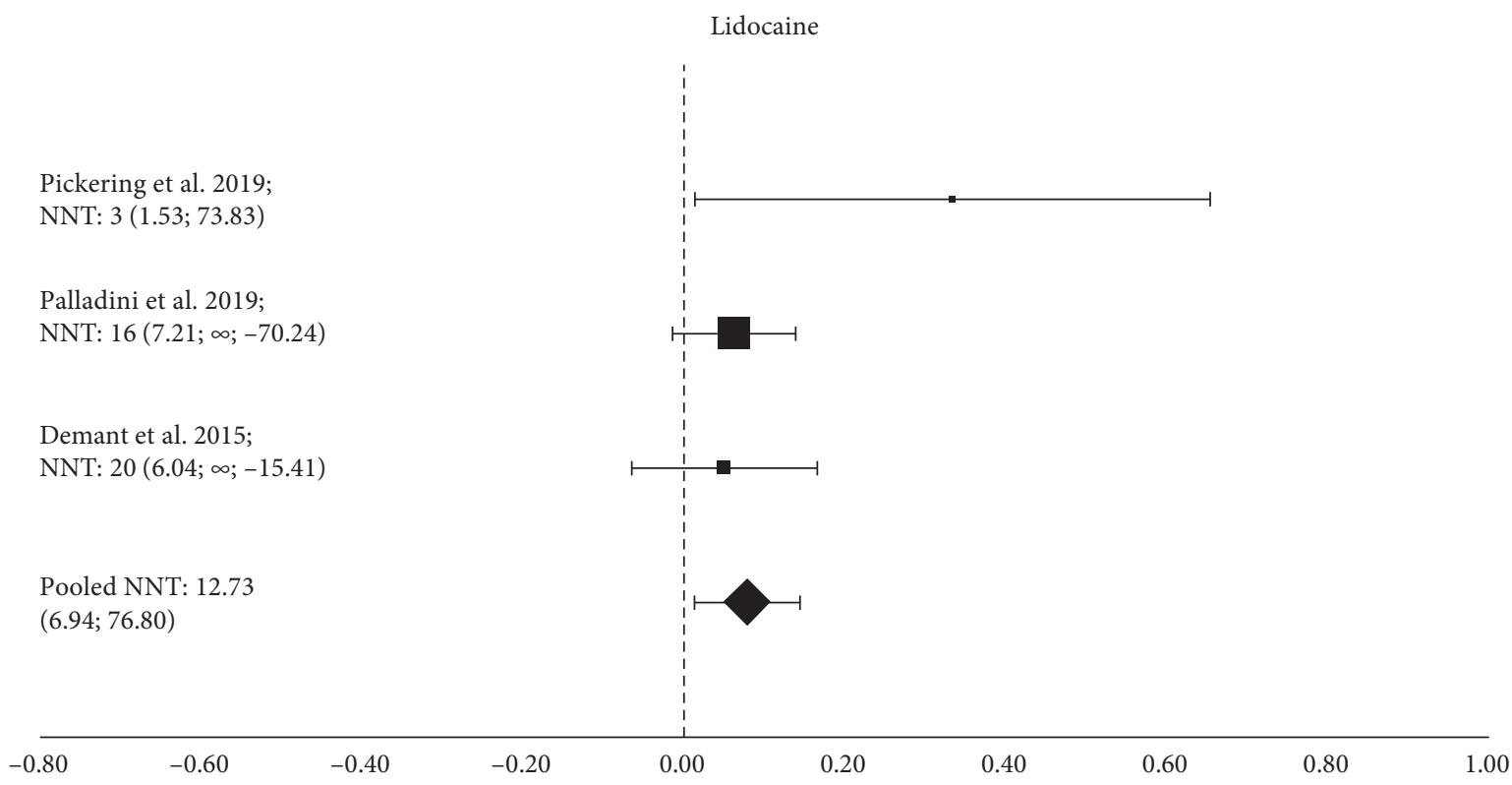

(d)

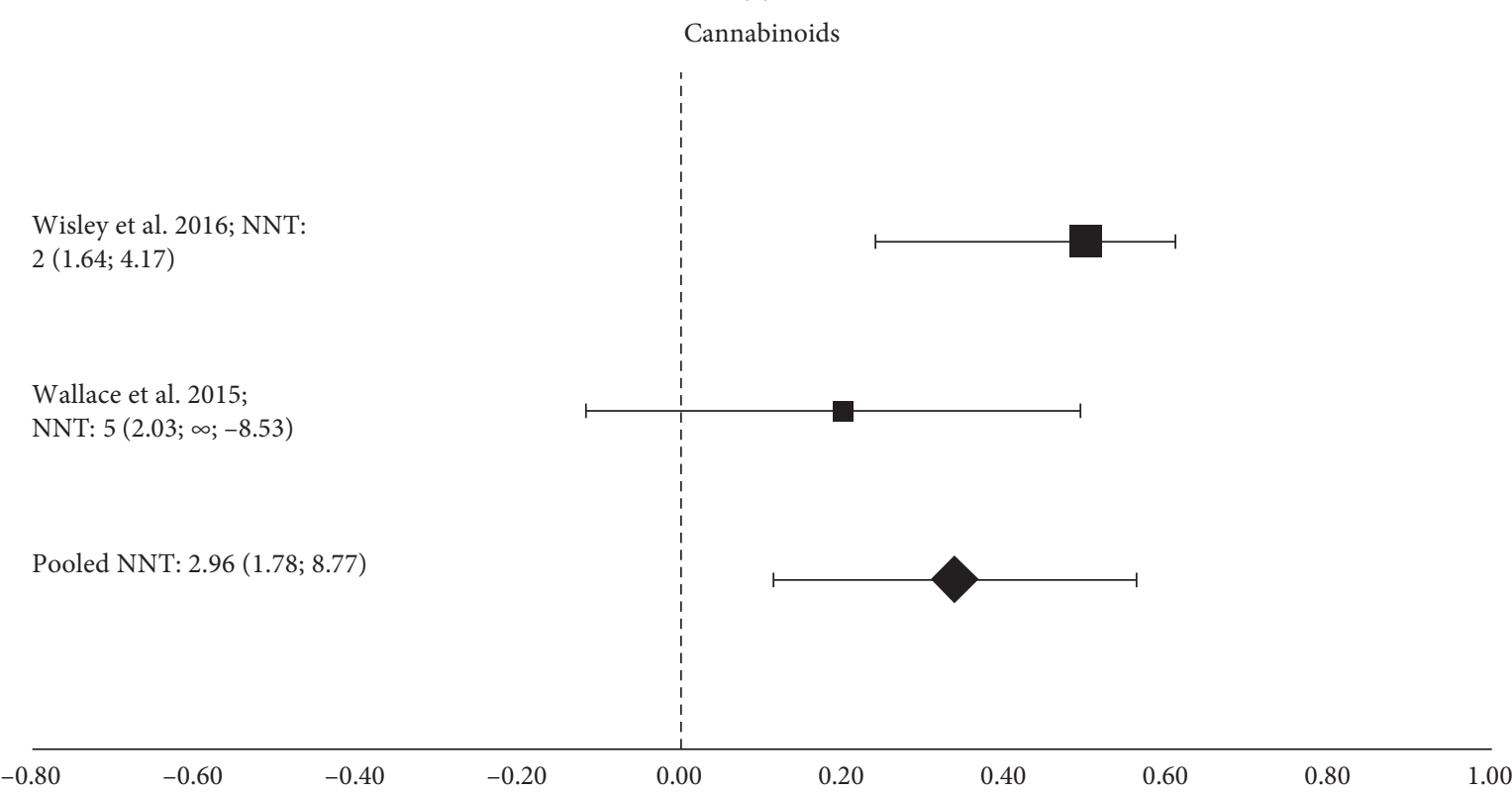

(e)

FIGURE 2: Forest plots of randomised controlled trials included in the metanalysis. Each value is expressed as mean NNT (95\% CI).

The CGRP receptor antibody, erenumab, is currently being compared with placebo in patients with trigeminal neuralgia (ClinicalTrials.gov Identifier: NCT04054024). Other drugs currently being tested in RCTs include $\mathrm{N}$-desmethylclobazam in patients with peripheral neuropathic pain (ClinicalTrials.gov Identifier: NCT04480164), clonidine, and pentoxifylline in different neuropathic pain conditions (ClinicalTrials.gov Identifier: NCT03342950), ziconotide in patients with severe refractory neuropathic pain (ClinicalTrials.gov Identifier: NCT03942848), and brivaracetam in spinal cord injury (ClinicalTrials.gov Identifier: NCT04379011).

\section{Discussion}

This manuscript provides an update to the 2015 NeuPSIG meta-analysis on neuropathic pain treatment [3]. We analysed 39 RCTs in our systematic review and included 16 trials in our meta-analysis.

We found that the combined NNT was $12.41(4.07 ; \infty$; -11.83 ) for antidepressants (venlafaxine XR and imipramine), $8.40(4.85$; 31.15) for gabapentinoids (pregabalin and mirogabalin), $4.64(2.59 ; 22.40)$ for selective sodium channel blockers, $12.73(6.94 ; 76.79)$ for lidocaine, and $2.96(1.78 ; 8.77)$ for cannabinoids (Figure 2). 
Admittedly, the low number of studies included in the meta-analysis may affect the external consistency of these NNT values.

We found poor trial outcomes even for first-line drugs such as antidepressants and pregabalin. These findings, consistent with those of the previous NeuPSIG metanalysis [3], may partly reflect the high placebo response and the lack of adequate diagnostic criteria for neuropathic pain. Another possible issue concerns the heterogeneity of patient phenotypes in clinical trials, which may underlie heterogeneous pathophysiological mechanisms and, thus, different drug responses. Recent clinical trials and post hoc analyses suggest that some drugs might be differentially effective in patients classified on the basis of their sensory phenotype $[23,45]$. In the study by Demant et al., the sodium channel blocker oxcarbazepine relieved peripheral neuropathic pain more efficaciously in patients with the irritable nociceptor phenotype, in which upregulation of sodium channels in nociceptors has been proposed as the pain-generating mechanism [23]. These data highlight the need for bettertargeted existing therapies and a mechanism-based personalised treatment of neuropathic pain.

Unexpectedly, in our meta-analysis, we found that antidepressants had a relatively high NNT, larger than that reported in the previous NeuPSIG meta-analysis. This finding, however, may merely reflect the inclusion of only two studies that tested the effect of venlafaxine XR and imipramine. We did not include any study on the efficacy of amitriptyline, the most widely used tricyclic antidepressant for neuropathic pain.

For pregabalin, we found a slightly larger combined NNT than that reported in the previous NeuPSIG metanalysis. The effect size of pregabalin and its clinical usefulness are currently sources of debate [46, 47]. Recent recommendations issued by the French chapter of the IASP and the French Society of Neurology [46] have proposed pregabalin as an alternative to gabapentin for second-line treatment, given that different studies $[3,48]$ have shown that gabapentin has a relatively large effect size and tolerability.

Our meta-analysis showed a large NNT for topical lidocaine (about 12). This finding is in line with previous large meta-analyses $[3,46]$. However, due to its excellent safety profile, topical lidocaine is commonly recommended as a first- or second-line drug, particularly in patients with localised neuropathic pain $[3,46]$.

We found a large effect $(\mathrm{NNT}=2.96)$ for cannabinoids. This finding is in contrast to the previous NeuPSIG recommendations, which provided a weak recommendation against the use of cannabinoids in patients with neuropathic pain [3]. Our finding on the efficacy of cannabinoids was probably affected by an overestimation of their effect due to the inclusion of only two studies. Hence, we believe that reliable evidence is still needed regarding the efficacy and safety of cannabinoids in patients with neuropathic pain.

In this meta-analysis, we have now included also selective Nav1.7 sodium channel blockers. We found a larger effect of new selective Nav1.7 sodium channel blockers than that of currently used neuropathic pain medications, including first-line drugs. Nav1.7 plays a key role in pain perception [49] and has a relatively specific expression in the peripheral nervous system, thus suggesting that selective Nav1.7 sodium channel blockers may have fewer side effects than currently available analgesics. Different studies are currently assessing the efficacy and safety of these new compounds in different neuropathic pain conditions. This research field is also supported by evidence of voltage-gated $\mathrm{Na}+$ channel variations in the pathogenesis of different neuropathic pain conditions [50-52]. The increasing availability of gene sequencing, combined with structural modelling and electrophysiological analysis of gene variants, may provide an opportunity to better target existing therapies. The anticonvulsant lacosamide, acting on Nav1.3, Nav1.7, and Nav1.8 channels, was tested in patients with Nav1.7-mutation-related small-fibre neuropathy, with promising findings in a subgroup of patients [33]. Labau and colleagues, using voltage-clamp recordings, found a preferential effect of lacosamide on Nav1.7 variants in patients who were responsive to lacosamide via a hyperpolarizing shift in the voltage dependence of both fast and slow inactivation and enhancement of use-dependent inhibition [53]. In addition, recent studies have shown that carbamazepine, at clinically achievable concentrations, acts via a new mode of action as an activation modulator of select mutant Nav1.7 channels [54, 55].

Another novel research field concerns the development of antibodies targeting the activity of human nerve growth factors. In a phase II trial in patients with postherpetic neuralgia and painful posttraumatic neuropathy [42], fulranumab did not significantly reduce pain in comparison with placebo. When subcutaneous and intravenous tanezumab was compared with placebo in patients with diabetic painful neuropathy and postherpetic neuralgia [43], pain relief was significantly higher with tanezumab than with placebo in only diabetic patients.

Among the new emerging drugs, ASP8477, a selective fatty acid amide hydrolase inhibitor, is under development. This drug was recently tested in patients with peripheral neuropathic pain and showed a good safety profile, though no significant pain relief as compared with placebo was found [40].

\section{Conclusions}

Our systematic review and meta-analysis, which included data from randomised controlled trials in patients with neuropathic pain, extend previous knowledge by demonstrating small effect sizes and/or large NNTs for all compounds used to treat neuropathic pain [2]. The modest efficacy of available treatments suggests the need for novel drug options. Drugs acting on new pain targets, including sodium channel isoform-specific antagonists and monoclonal antibodies, are currently under development. In particular, the new selective Nav1.7 sodium channel blockers might be an effective treatment option for a selected population $[49,50]$. 


\section{Data Availability}

All data generated or analysed during this study are included in this published article.

\section{Conflicts of Interest}

The authors declare no conflicts of interest.

\section{References}

[1] D. Bouhassira and N. Attal, "Emerging therapies for neuropathic pain: new molecules or new indications for old treatments?" Pain, vol. 159, no. 3, pp. 576-582, 2018.

[2] P. Ciaramitaro, G. Cruccu, G. Cruccu et al., "A delphi consensus statement of the neuropathic pain special interest group of the Italian neurological society on pharmacoresistant neuropathic pain," Neurological Sciences, vol. 40, no. 7, pp. 1425-1431, 2019.

[3] N. B. Finnerup, N. Attal, S. Haroutounian et al., "Pharmacotherapy for neuropathic pain in adults: a systematic review and meta-analysis," The Lancet Neurology, vol. 14, no. 2, pp. 162-173, 2015.

[4] A. R. Jadad, R. A. Moore, D. Carroll et al., "Assessing the quality of reports of randomized clinical trials: is blinding necessary?" Controlled Clinical Trials, vol. 17, no. 1, pp. 1-12, 1996.

[5] M. Borenstein, L. V. Hedges, J. P. T. Higgins, and H. R. Rothstein, "A basic introduction to fixed-effect and random-effects models for meta-analysis," Research Synthesis Methods, vol. 1, no. 2, pp. 97-111, 2010.

[6] N. Majdinasab, H. Kaveyani, and M. Azizi, "A comparative double-blind randomized study on the effectiveness of Duloxetine and Gabapentin on painful diabetic peripheral polyneuropathy," Drug Design, Development and Therapy, vol. 13, pp. 1985-1992, 2019.

[7] N. Farshchian, A. Alavi, S. Heydarheydari, and N. Moradian, "Comparative study of the effects of venlafaxine and duloxetine on chemotherapy-induced peripheral neuropathy," Cancer Chemotherapy and Pharmacology, vol. 82, no. 5, pp. 787-793, 2018.

[8] S. Brown, B. Johnston, K. Amaria et al., "A randomized controlled trial of amitriptyline versus gabapentin for complex regional pain syndrome type I and neuropathic pain in children," Scandinavian Journal of Pain, vol. 13, no. 1, pp. 156-163, 2016.

[9] J. S. Richards, C. H. Bombardier, C. S. Wilson et al., "Efficacy of venlafaxine XR for the treatment of pain in patients with spinal cord injury and major depression: a randomized, controlled trial," Archives of Physical Medicine and Rehabilitation, vol. 96, no. 4, pp. 680-689, 2015.

[10] J. V. Holbech, F. W. Bach, N. B. Finnerup, K. Brøsen, T. S. Jensen, and S. H. Sindrup, "Imipramine and pregabalin combination for painful polyneuropathy," Pain, vol. 156, no. 5, pp. 958-966, 2015.

[11] M. Baba, N. Matsui, M. Kuroha, Y. Wasaki, and S. Ohwada, "Mirogabalin for the treatment of diabetic peripheral neuropathic pain: a randomized, double-blind, placebo-controlled phase III study in Asian patients," Journal of Diabetes Investigation, vol. 10, no. 5, pp. 1299-1306, 2019.

[12] J. Jiang, Y. Li, Q. Shen et al., "Effect of pregabalin on radiotherapy-related neuropathic pain in patients with head and neck cancer: a randomized controlled trial," Journal of Clinical Oncology, vol. 37, no. 2, pp. 135-143, 2019.
[13] J. Kato, N. Matsui, Y. Kakehi, E. Murayama, S. Ohwada, and M. Sugihara, "Mirogabalin for the management of postherpetic neuralgia: a randomized, double-blind, placebocontrolled phase 3 study in Asian patients," Pain, vol. 160, no. 5, pp. 1175-1185, 2019.

[14] C. L. Huffman, J. N. Goldenberg, J. Weintraub et al., "Efficacy and safety of once-daily controlled-release pregabalin for the treatment of patients with postherpetic neuralgia," The Clinical Journal of Pain, vol. 33, no. 7, pp. 569-578, 2017.

[15] Q. Liu, H. Chen, L. Xi et al., "A randomized, double-blind, placebo-controlled trial to evaluate the efficacy and safety of pregabalin for postherpetic neuralgia in a population of Chinese patients," Pain Practice, vol. 17, no. 1, pp. 62-69, 2017.

[16] D. Merante, R. Julio, S. Uma, F. Karen, H. Ching, and V. Aaron, "DS-5565-A-U201 US phase 2 study investigators. Efficacy of mirogabalin (DS-5565) on patient-reported pain and sleep interference in patients with diabetic neuropathic pain: secondary outcomes of a phase II proof-of-concept study," Pain Medicine, vol. 18, no. 11, pp. 2198-2207, 2017.

[17] P. Raskin, C. Huffman, L. Yurkewicz et al., "Pregabalin in patients with painful diabetic peripheral neuropathy using an NSAID for other pain conditions," The Clinical Journal of Pain, vol. 32, no. 3, pp. 203-210, 2016.

[18] R. Freeman, M. S. Wallace, M. Sweeney, and M. M. Backonja, "Relationships among pain quality, pain impact, and overall improvement in patients with postherpetic neuralgia treated with gastroretentive gabapentin," Pain Medicine, vol. 16, no. 10, pp. 2000-2011, 2015.

[19] M. Palladini, I. Boesl, S. Koenig, B. Buchheister, and N. Attal, "Lidocaine medicated plaster, an additional potential treatment option for localized post-surgical neuropathic pain: efficacy and safety results of a randomized, placebo-controlled trial," Current Medical Research and Opinion, vol. 35, no. 5, pp. 757-766, 2019.

[20] G. Pickering, M. Voute, N. Macian, H. Ganry, and B. Pereira, "Effectiveness and safety of 5\% lidocaine-medicated plaster on localized neuropathic pain after knee surgery: a randomized, double-blind controlled trial," Pain, vol. 160, no. 5, pp. 1186-1195, 2019.

[21] Y.-C. Kim, A. M. Castañeda, C.-s. Lee, H.-S. Jin, K. S. Park, and J. Y. Moon, "Efficacy and safety of lidocaine infusion treatment for neuropathic pain," Regional Anesthesia and Pain Medicine, vol. 43, no. 4, pp. 415-424, 2018.

[22] H. Liu, F. Lu, D. Zhou et al., "The analgesic and emotional response to intravenous lidocaine infusion in the treatment of postherpetic neuralgia," The Clinical Journal of Pain, vol. 34, no. 11, pp. 1025-1031, 2018.

[23] D. T. Demant, K. Lund, N. B. Finnerup et al., "Pain relief with lidocaine $5 \%$ patch in localized peripheral neuropathic pain in relation to pain phenotype: a randomised, double-blind, and placebo-controlled, phenotype panel study," Pain, vol. 156, no. 11, pp. 2234-2244, 2015.

[24] P. D. Gavin, L. Tremper, A. Smith, G. Williams, and C. Brooker, "Transdermal oxycodone patch for the treatment of postherpetic neuralgia: a randomized, double-blind, controlled trial," Pain Management, vol. 7, no. 4, pp. 255-267, 2017.

[25] F. K. Rigo, G. Trevisan, M. C. Godoy et al., "Management of neuropathic chronic pain with methadone combined with ketamine: a randomized, double blind, active-controlled clinical trial," Pain Physician, vol. 20, no. 3, pp. 207-215, 2017.

[26] R. W. Simpson and J. H. Wlodarczyk, "Transdermal buprenorphine relieves neuropathic pain: a randomized, double- 
blind, parallel-group, placebo-controlled trial in diabetic peripheral neuropathic pain," Diabetes Care, vol. 39, no. 9, pp. 1493-1500, 2016.

[27] I. Gilron, D. Tu, R. R. Holden, A. C. Jackson, and D. DuMerton-Shore, "Combination of morphine with nortriptyline for neuropathic pain," Pain, vol. 156, no. 8, pp. 1440-1448, 2015.

[28] S. Schimrigk, M. Marziniak, C. Neubauer, E. Maria Kugler, G. Werner, and D. Abramov-Sommariva, "Dronabinol is a safe long-term treatment option for neuropathic pain patients," European Neurology, vol. 78, no. 5-6, pp. 320-329, 2017.

[29] G. van Amerongen, K. Kanhai, A. C. Baakman et al., "Effects on spasticity and neuropathic pain of an oral Formulation of $\Delta$ 9-tetrahydrocannabinol in patients with progressive multiple sclerosis," Clinical Therapeutics, vol. 40, no. 9, pp. 1467-1482, 2018.

[30] B. Wilsey, T. D. Marcotte, R. Deutsch, H. Zhao, H. Prasad, and A. Phan, "An exploratory human laboratory experiment evaluating Vaporized cannabis in the treatment of neuropathic pain from spinal cord injury and disease," The Journal of Pain, vol. 17, no. 9, pp. 982-1000, 2016.

[31] D. Turcotte, M. Doupe, M. Torabi et al., "Nabilone as an adjunctive to gabapentin for multiple sclerosis-induced neuropathic pain: a randomized controlled trial," Pain Medicine, vol. 16, no. 1, pp. 149-159, 2015.

[32] M. S. Wallace, T. D. Marcotte, A. Umlauf, B. Gouaux, and J. H. Atkinson, "Efficacy of inhaled cannabis on painful diabetic neuropathy," The Journal of Pain, vol. 16, no. 7, pp. 616-627, 2015.

[33] B. T. A. de Greef, J. G. J. Hoeijmakers, M. Geerts et al., "Lacosamide in patients with Nav1.7 mutations-related small fibre neuropathy: a randomized controlled trial," Brain, vol. 142, no. 2, pp. 263-275, 2019.

[34] A. McDonnell, S. Collins, Z. Ali et al., "Efficacy of the Nav1.7 blocker PF-05089771 in a randomised, placebo-controlled, double-blind clinical study in subjects with painful diabetic peripheral neuropathy," Pain, vol. 159, no. 8, pp. 1465-1476, 2018.

[35] J. M. Zakrzewska, J. Palmer, V. Morisset et al., "Safety and efficacy of a Nav1.7 selective sodium channel blocker in patients with trigeminal neuralgia: a double-blind, placebocontrolled, randomised withdrawal phase 2a trial," The Lancet Neurology, vol. 16, no. 4, pp. 291-300, 2017.

[36] N. Price, R. Namdari, J. Neville et al., "Safety and efficacy of a topical Sodium Channel inhibitor (TV-45070) in patients with postherpetic neuralgia (PHN)," The Clinical Journal of Pain, vol. 33, no. 4, pp. 310-318, 2017.

[37] K. Kulkantrakorn, A. Chomjit, P. Sithinamsuwan, T. Tharavanij, J. Suwankanoknark, and P. Napunnaphat, " $0.075 \%$ capsaicin lotion for the treatment of painful diabetic neuropathy: a randomized, double-blind, crossover, placebocontrolled trial," Journal of Clinical Neuroscience: Official Journal of the Neurosurgical Society of Australasia, vol. 62, pp. 174-179, 2019.

[38] N. Kerckhove, B. Pereira, S. Soriot-Thomas et al., "Efficacy and safety of a T-type calcium channel blocker in patients with neuropathic pain: a proof-of-concept, randomized, doubleblind and controlled trial," European Journal of Pain, vol. 22, no. 7, pp. 1321-1330, 2018.

[39] Vitamin E in Neuroprotection Study (VENUS) Investigators, C. P. Hor, W. Y. Fung, H. A. Ang, S. C. Lim et al., "Efficacy of oral mixed tocotrienols in diabetic peripheral neuropathy: a randomized clinical trial," JAMA Neurology, vol. 75, no. 4, pp. 444-452, 2018.

[40] D. Bradford, A. Stirling, E. Ernault et al., "The MOBILE studya phase IIa enriched enrollment randomized withdrawal trial to assess the analgesic efficacy and safety of ASP8477, a fatty acid amide hydrolase inhibitor, in patients with peripheral neuropathic pain," Pain Medicine, vol. 18, no. 12, pp. 2388-2400, 2017.

[41] S. R. Andresen, J. Bing, R. M. Hansen et al., "Ultramicronized palmitoylethanolamide in spinal cord injury neuropathic pain: a randomized, double-blind, placebo-controlled trial," Pain, vol. 157, no. 9, pp. 2097-2103, 2016.

[42] H. Wang, G. Romano, M. Fedgchin et al., "Fulranumab in patients with pain associated with postherpetic neuralgia and postraumatic neuropathy," The Clinical Journal of Pain, vol. 33, no. 2, pp. 99-108, 2017.

[43] C. Bramson, D. N. Herrmann, W. Carey et al., "Exploring the role of tanezumab as a novel treatment for the relief of neuropathic pain," Pain Medicine, vol. 16, no. 6, pp. 1163-1176, 2015.

[44] S. U. Ahmed, Y. Zhang, L. Chen et al., "Effect of 1.5\% topical diclofenac on clinical neuropathic pain," Anesthesiology, vol. 123, no. 1, pp. 191-198, 2015.

[45] D. Bouhassira, S. Wilhelm, A. Schacht et al., "Neuropathic pain phenotyping as a predictor of treatment response in painful diabetic neuropathy: data from the randomized, double-blind, COMBO-DN study," Pain, vol. 155, no. 10, pp. 2171-2179, 2014.

[46] X. Moisset, B. Pereira, D. Bouhassira, and N. Attal, "Pregabalin: a better neuropathic pain treatment in rodents than in humans," Pain, vol. 161, no. 10, pp. 2425-2427, 2020.

[47] X. Moisset, D. Bouhassira, J. Avez Couturier et al., "Pharmacological and non-pharmacological treatments for neuropathic pain: systematic review and French recommendations," Revue Neurologique, vol. 176, no. 5, pp. 325-352, 2020.

[48] K. Robertson, L. A. G. Marshman, D. Plummer, and E. Downs, "Effect of gabapentin vs pregabalin on pain intensity in adults with chronic sciatica," JAMA Neurology, vol. 76, no. 1, pp. 28-34, 2019.

[49] D. L. Bennett, A. J. Clark, J. Huang, S. G. Waxman, and S. D. Dib-Hajj, "The role of voltage-gated sodium channels in pain signaling," Physiological Reviews, vol. 99, no. 2, pp. 1079-1151, 2019.

[50] G. Di Stefano, J.-H. Yuan, G. Cruccu, S. G. Waxman, S. D. Dib-Hajj, and A. Truini, "Familial trigeminal neuralgia a systematic clinical study with a genomic screen of the neuronal electrogenisome," Cephalalgia, vol. 40, no. 8, pp. 767-777, 2020.

[51] I. Blesneac, A. C. Themistocleous, C. Fratter et al., "Rare NaV1.7 variants associated with painful diabetic peripheral neuropathy," Pain, vol. 159, no. 3, pp. 469-480, 2018.

[52] C. G. Faber, J. G. J. Hoeijmakers, H.-S. Ahn et al., "Gain of function NaV1.7 mutations in idiopathic small fiber neuropathy," Annals of Neurology, vol. 71, no. 1, pp. 26-39, 2012.

[53] J. I. R. Labau, M. Estacion, B. S. Tanaka et al., "Differential effect of lacosamide on Nav1.7 variants from responsive and non-responsive patients with small fibre neuropathy," Brain, vol. 143, no. 3, pp. 771-782, 2020.

[54] T. Z. Fischer, E. S. Gilmore, M. Estacion et al., “A novel Nav1.7 mutation producing carbamazepine-responsive erythromelalgia," Annals of Neurology, vol. 65, no. 6, pp. 733-741, 2009. 
[55] Y. Yang, T. Adi, P. R. Effraim, L. Chen, S. D. Dib-Hajj, and S. G. Waxman, "Reverse pharmacogenomics: carbamazepine normalizes activation and attenuates thermal hyperexcitability of sensory neurons due to Nav 1.7 mutation I234T," British Journal of Pharmacology, vol. 175, no. 12, pp. 22612271, 2017. 